\title{
The Need to Study Within-Person Processes in Psychotherapy: A Discussion of Alliance Studies
}

\author{
Asle Hoffart*
}

Research Institute, Modum Bad, Vikersund and Department of Psychology, University of Oslo, Norway

Received: February 22, 2014; Accepted: April 07, 2014; Published: April 09, 2014

*Corresponding author: Asle Hoffart, Department of Psychology, University of Oslo, Modum Bad, Vikersund, Norway, E-mail: asle.hoffart@modum-bad.no

\begin{abstract}
Research on the alliance has mainly focused between-patient data (e.g., how differences in alliance among patients are related to outcome for a patient compared to other patients). However, this level of analysis partly misses its target because psychotherapy theories and therapists focus primarily on within-patient processes (e.g., whether change in a client's alliance during the course of therapy may lead to a reduction of symptoms in that client). So far, the ability to separate these effects has not been fully capitalized upon in alliance research. Notable exceptions are the studies of Tasca and Lampard [1] and Falkenström et al. [2] and our own study Hoffart et al. [3]. The purpose of the present short communication is to present and discuss these studies, with particular emphasis on our own study.
\end{abstract}

Keywords: Alliance; Process research; Within-person relationships

\section{Introduction}

According to the most widely accepted trans-theoretical model, alliance is composed of agreement about the tasks of therapy, agreement about the goals of therapy, and the emotional bond between patient and therapist [4] . A stronger therapeutic alliance has been found to be associated with better outcomes across a variety of treatment approaches and mental health problems [5-7]. This research has mainly focused betweenpatient data, that is, how differences in alliance among patients are related to differences in outcome among the patients. However, this level of analysis partly misses its target because psychotherapy theories and therapists focus primarily on within-patient processes. For instance, a therapist is interested in whether a repair of a rupture in the alliance with a patient at a certain point in therapy [8] may lead to subsequent reduction of that patient's symptoms.

An example from the medical field illustrates the necessity to distinguish the between- and within-person levels of effect. It has been shown that an individual is more likely to experience a heart attack while exercising than when he/she is not (the within-person effect). Still, people who exercise tend to have a lower risk of heart attack (the between-person effect). Thus, the effects are opposite to each other, and generalizing findings at one level to the other would be an error of inference. These issues are likely to be relevant in most areas of psychological research.
The typical alliance data, collected once early in therapy, or occasionally during therapy, are poorly suited for evaluating within-person processes [9]. Only repeated measures data allow for the proper disaggregation of between-person and withinperson effects $[9,10]$. When a set of measures is collected at a single point of time from multiple individuals, the resulting data provide information only about between-person relationships. In contrast, when a set of measures are collected at multiple points in time from multiple individuals, the resulting data contain information about both between-person and withinperson differences. Such data must be carefully specified to avoid confounding the two sources of variability.

A disaggregation of the between-person and within-person variance components of a predictor not only allows the study of within-person processes separated from between-person effects, but also is able to examine cross-level interactions of betweenand within-person effects. For instance, the effect of reporting more alliance than expected for a particular patient may matter more for patients who have lower alliance in general. When the general (between-person) level of bond is low, for example when the patient has low trust that the therapist wants the best for him/her and is therefore preoccupied with this issue, a certain increase of this trust in a particular session might be a valued event with an immediate effect on symptoms. On the other hand, when the patient's trust is high already and not an issue for him/ her, the same increase would probably have less consequences.

So far, the ability to separate these effects has not been fully capitalized upon in alliance research. Notable exceptions are the studies of Tasca and Lampard [1] and Falkenström et al. [2] and our own study Hoffart et al. [3]. The purpose of the present short communication is to present and discuss these studies, with particular emphasis on our own study.

\section{Discussion of studies involving disaggregated alliance score}

Tasca and Lampard [1] examined the reciprocal influence of change in alliance to the group and change in urge to restrict food intake in eating-disordered individuals $(\mathrm{N}=234)$ attending a group-based day treatment. The California Psychotherapy Alliance Scales-Group Patient Version (CALPAS-G) [11] was the 
measure of alliance to patients in the group. The CALPAS-G is based on Bordin's [4] pantheoretical definition of the alliance and includes 12 items describing agreement on goals, agreement on tasks, and bond. A mean item total score is calculated. Using latent change score modeling, in which between- and withinperson components of both the predictor and outcome variables are separated, Tasca and Lampard obtained evidence for a reciprocal influence of alliance to the patient group and outcome on a week-by-week time level over the course of the 9 first weeks in the program.

Falkenström [2] studied the reciprocal relationship of alliance and outcome in a sample of 646 patients in primary care psychotherapy. Alliance was measured by the Working Alliance Inventory-Short Revised (WAI-SR) [12] it is also based on Bordin's definition of the alliance and consists of three components: agreement about goals, agreement about tasks, and bond. The mean item total score was used in this study. Using the disaggregation methods in multilevel models proposed by Curran and Bauer [9], Falkenström et al. also found evidence for a reciprocal causal model of alliance and outcome on a sessionby-session level.

The main purpose of our study [3] was to examine the role of alliance components in the process of therapeutic change in PTSD patients. The patients received either standard prolonged exposure [13] which includes imagery exposure (IE) to the traumatic memory, or modified prolonged exposure, where imagery rescripting [14]) replaced IE as the imagery component of prolonged exposure in a 10 week residential program. The WAI-SR was used as a measure of alliance and the 4 item goal (e.g., "The therapist and I are working towards mutually agreed upon goals"), 4 item task (e.g., "I feel that the things I do in therapy will help me to accomplish the changes that I want"), and 4 item bond (e.g., "I feel that the therapist appreciates me") subscales were used in the analyses. The mean age of the patients $(\mathrm{N}=65$; 38 women and 27 men) was 45.2 years $(S D=9.7)$ and the mean length of time since the main trauma was 17.5 years $(S D=13.3)$. The patients had experienced various traumas, for over half of them the main trauma was a prolonged and/or repeated event. They were assessed repeatedly (weekly) on alliance and PTSD symptom measures, allowing us to separate the variance related to individual differences (between-person component) at the start of treatment from variance related to the intra-individual process of change during treatment (within-person component). Like in the study of Falkenström et al., the centering method of detrending was used to disaggregate the within-person and between-person variability in the predictors [9].

We hypothesized that the task and bond components should be particularly influential on outcome in traumafocused treatments of PTSD because of the demands put on both the patient and the therapist. In prolonged exposure, the patient is asked to approach what has evoked the most anxiety and distress. During the IE part of prolonged exposure, also the therapist is confronted with aversive information, which may evoke emotional responses he or she cannot express and consequently induce therapist feelings of powerlessness. The strains put on the patient and the therapist potentially require an agreement about these tasks, which suggests that both the patient and the therapist understand and accept the treatment rationale and believe that the treatment is an effective approach to reduce symptoms. Furthermore, the traumatic experience and its aftermaths often involve helplessness, shame, guilt, and anger reactions that are difficult to reveal to another person [15]. Many clients also fear that IE to the trauma memory will lead to loss of control and even insanity. All this requires a development of a bond, in which the patient trusts that the therapist understands, cares for, and accepts him/her, and believes the therapist is able to help the patient regulate strong emotions [16]. Actually, the hypothesis of a negative within-person effect of the components agreement about the tasks of therapy and bond on subsequent PTSD symptoms was supported for the task component. That is, when the task score for a given patient was higher than was expected for that patient, the subsequent symptom score was lower than was expected for him/her, explaining about $4 \%$ of the outcome variance. In any event, this finding goes beyond previous research in the PTSD treatment field by indicating that time-specific change in a person's task agreement during therapy is related to this person's subsequent change in PTSD symptoms. On a more general level, this finding supports and extends those of Tasca and Lampard [1] and Falkenström et al. [2], who found a within-person relationship between overall alliance and subsequent symptoms but in different treatments and patient populations.

We also hypothesized that, due to the empowering and relieving features of IR compared to IE, the influence of task agreement and bond on subsequent PTSD symptoms would be weaker in IR than in IE. In IR, the patient's Current Self is - after an initial imagery exposure phase - invited to enter the imagery at the worst moment of the trauma, bring the situation to a solution (e.g., overpower a perpetrator), and then interact with the Traumatized Self back-then. The patient's anger is used as a resource in overpowering perpetrators and the Current SelfTraumatized Self interaction stimulates the development of self-compassion instead of shame, guilt, and self-critique. The empowering and relieving features of IR may put less strain on the patient and the therapists by making them feel less helpless and distressed compared to IE and thus help them both to engage in imagery work. Actually, our results indicated that the withinperson relationship between task agreement and outcome is dependent on the specific therapy form. As we hypothesized, this relationship was stronger in IE than in IR.

On the other hand, within-person changes in PTSD symptoms did not predict subsequent task agreement and bond. That is, time-specific change in a person's symptoms during therapy was not related to this person's subsequent change in task and bond. This finding is at odds with those of Tasca and Lampard [1] and Falkenström et al. [2] who found a bi-directional relationship between alliance and symptoms. A conspicuous difference between these studies and ours is that we used standardized, highly structured and manual-based procedures. One may speculate that patients' belief in and agreement to such procedures are less influenced by symptom variations than is their agreement to less standardized and clearly defined 
procedures. Also the possibly lesser focus on the bonding and affective elements of the relationship in our therapies may have influenced the results [17].

Our hypotheses about a between-person effect of initial task agreement and bond was supported. Initial task and bond scores predicted a steeper negative slope of PTSD symptoms. These findings are consistent with most findings in alliance research [7] that early alliance predicts the further course of symptoms.

We expected that task agreement and bond would be of greater concern for those who had a lower individual level and thus be more influential in these persons' process of change, but - in fact - the within-person effect of task scores on subsequent PTSD symptoms was stronger in those with a higher initial task agreement. Future research must show whether this was a chance finding or not. However, if this effect is replicated, it would suggest a double drawback for a patient having a low initial agreement about the tasks of therapy. First, the patient would experience less overall improvement over the course of therapy, and, second, greater than expected levels of agreement during the process of therapy for that patient would not be as effective.

We also explored the role of the alliance component goal agreement. As expected, this component was unrelated to symptom change. The within-person component of goal agreement also had less variance than the within-person component of task agreement, and this difference may have contributed to the differential findings. Our results are consistent with Horvath et al. [7], who found - on the between-person level that the agreement on tasks was a superior predictor of outcome than were either bond or agreement on goals.

\section{Strengths of the multi-level approach}

Alliance and PTSD symptoms were assessed weekly and adequate methods were utilized to separate the within-person and between-person effects of the time-varying predictors in the applied multilevel models. Thus, we could study withinperson relationships over the course of therapy, which are of particular relevance for psychotherapy theories. This is because therapy theories concern such relationships, that is, how change in a process variable relates to subsequent change in an outcome variable. Such knowledge directly informs therapists what process variables need to be affected to achieve patient improvement. By contrast, knowledge of between-person relationships - one patient having a low initial alliance and poor outcome and another having a high initial alliance and good outcome - does not imply that an increase in the first patient's alliance would lead to better outcome for that patient. Thus, relationships established on a between-person level do not imply that the same relationships hold on a within-person level. For instance, the relationship between bond and outcome obtained in the present study on the between-person level was not replicated on the within-person level. A further advantage of properly separating the between- and within-person components of a time-varying predictor is the possibility to examine cross-level interactions of within- and between-person effects. For therapists, how between-person differences in for example alliance or selfconcept moderate within-person relationships over the course of therapy is more directly relevant than the correlations of these differences with overall outcome. Such moderating knowledge inform therapists under what conditions (e.g., low sense of self relative to other patients) certain within-person change processes are working (e.g., higher than usual sense of self on a given time-point predicts lower than usual PTSD symptoms) and should be focused. A further advantage of studying within-person relationships between process and outcome is the possibility to identify reciprocal or even reversed causality between process and outcome. The RCT design of our study, where patients were randomized to two empirically based imagery methods, allowed us to study the moderating influence of therapy form on the within-person relationships.

\section{Limitations of the multi-level approach}

It is important to acknowledge the limitations of the multilevel within- and between-effects approach. Both these effects concern linear relationships between process and outcome variables. It is assumed that more of a process phenomenon will produce better therapeutic outcomes. However, such an assumption is dubious in many research contexts [17]. For instance, it is not appropriate when studying the mutual influence of therapists and patients during sessions, which concerns how the probability of certain behaviors on the part of one of them relates to the probability of certain subsequent behaviors on the part of the other. Sequence analysis is appropriate for analyzing such questions [8]. Neither is the approach appropriate for studying significant but infrequent phenomena in therapy [18]. The Interpersonal Process Recall interview is one method used to study significant events in therapy [19]. Finally, statistical relationships only depict regularities between abstract features and do not explain how a certain effect comes about, that is, the mechanism of change. Kazdin [20] defines a mechanism as the process or events that are responsible a change. For instance, the mechanisms of the relationships between alliance, engagement in exposure, and change in PTSD symptoms may be elucidated by Perceptual Control Theory (PCT) [21]. PCT proposes that all actions are goal-serving attempts to control one's own experiences. Both the patient and the therapist have many goals that are important when they are interacting. For instance, the intrusions of the PTSD patient may represent a survival goal to develop a plan of processing and action to use when faced with threat in the future [22]. The patient may also have the conflicting goal of avoiding the trauma memory because of the distress associated with it and/or because of fear of going crazy if engaging too much in it. He/she may as well have the goal of disguising his/her trauma-related emotions for the therapist. The therapist, on the other hand, may have the goals of promoting the patients free expression of experience in therapy and of guiding him/her through IE to the trauma memory. Therapists who formulate problems within the relationship as mismatches between their goals and those of the client embody a good therapeutic relationship/alliance. The sensitive negotiation of goals - where what happens is increasingly brought in conjunction with the participants goals - involves increasing agreement about the 
tasks of therapy. In turn, higher task agreement leads the patient to engage more in IE and therefore have a better outcome. PCT may also be used to understand how exposure works [23]. Such mechanistic relationships needs to be concretely modeled and testing of them involves simulations (for instance, by use of a computer) to see whether the expected outcome is actually produced [23]. Still, mechanistic models lead to predictions about statistical relationships on an aggregated level (e.g., between task agreement a session and subsequent PTSD symptoms) and these predictions may be tested by use of the multilevel approach presented here.

\section{Research implications}

The results of the three available studies of within-person relationships of alliance and symptoms over the course of therapy support that patients' alliance - both to the therapist and to the group of fellow patients - influences symptoms. The results are equivocal with respect to the reciprocal influence of symptoms on alliance. The reviewed studies invite an increased focus on within-person relationships in alliance research. In highly structured therapies like those of our study and cognitive therapy of depression Webb et al. [24], symptomatic improvement is supposed to result from the relatively specific tasks of these therapies. Agreement about tasks may therefore be particularly important in such therapies. Future studies should investigate the within-person relationships between alliance components and outcome across therapy forms, formats (e.g., individual vs. group therapy) and type and severity of disorders. Furthermore, studies of within-person relationships between therapy events/ therapist actions and alliance components are needed. Such correlational studies should be complemented with sequence analyses of therapy interactions and studies of mechanistic models for how interactions promote alliance.

\section{Clinical implications}

The results of our study must be interpreted with the reservation that the manualised form of treatment and the unusual residential setting may not generalize to practice in ordinary clinical settings. The fact that there were within-person variations in alliance in all three studies indicates that the relationships in therapy are not fixed once and for all at the start of therapy. It is rather a fluctuating and emergent property of the interaction during the sessions. The presented within-person results make a firm basis for the recommendation to monitor, increase and restore decreases of agreement about therapy tasks over the course of IE or IR within prolonged exposure for PTSD patients. They also suggest that addressing agreement about the tasks of therapy is particularly important in IE compared to IR. Given that these exposure methods consist of confronting the feared trauma memory and feared external situations, agreeing to their use based on an understanding of the rationale for these methods, a belief in their efficacy, and a sensible process of goal negotiation, seems paramount. On the other hand, the results do not imply an increased focus on the agreement about goals of therapy and bond components of alliance over the course of these treatments. The presented between-person results may inform therapists using prolonged exposure for PTSD that low initial task agreement and bond signal a poorer outcome of therapy. Unfortunately, because the cross-level interaction between interindividual and within-individual task agreement was contrary to the hypothesis, clinical implications cannot be drawn from this finding.

\section{References}

1. Tasca GA, Lampard AM (2012) Reciprocal influence of alliance to the group and Outcome in day treatment for eating disorders. Journal of Counseling Psychology 59: 507-517.

2. Falkenström F, Granström F, Holmqvist R (2013) Therapeutic alliance predicts symptomatic improvement session by session. Journal of Counseling Psychology Advance online publication 60(3): 317-328.

3. Hoffart A, Øktedalen T, Formo Langkaas T, Wampold, BE (2013) Alliance and outcome in varying imagery procedures for PTSD: A study of within-person processes. Journal of Counseling Psychology 60(4): 471-472.

4. Bordin ES (1979) The generalizability of the psychoanalytic concept of the working alliance. Psychotherapy 16: 252-260.

5. Del Re AC, Flückiger C, Horvath AO, Symonds D, Wampold BE (2012) Therapist effects in the therapeutic alliance-outcome relationship: A restricted-maximum likelihood meta-analysis. Clinical Psychology Review 32(7): 642-649.

6. Flückiger C, Del Re AC, Wampold BE, Symonds D, Horvath AO (2012) Howcentral is the alliance in psychotherapy A multilevel longitudinal meta-analysis. Journal of Counseling Psychology 59(1): 10-17.

7. Horvath AO, Del Re AC, Flückiger C, SymondsD (2011) Alliance in individual psychotherapy. Psychotherapy 48(1): 9-16.

8. Safran JD, Muran JC (1996) The resolution of ruptures in the therapeutic alliance. Journal of Consulting and Clinical Psychology 64(3): 447-48.

9. Curran PJ, Bauer DJ (2011) The disaggregation of within-person and between-person effects in longitudinal models of change. Annual Review of Psychology 62: 583-619.

10. Hoffman L, Sawski RS (2009) Persons as Contexts Evaluating Between-Person and Within-Person Effects in Longitudinal Analysis. Research in Human Development 6(2-3): 97-120.

11. Gaston L, Marmar CR (1993) Manual of the California Psychotherapy Alliance Scales (CALPAS). Unpublished manuscript, Department of Psychiatry, McGill University, Montreal, Quebec, Canada.

12. Hatcher RL, Gillaspy JA(2006) Development and validation of a short version of the Working Alliance Inventory. Psychotherapy Research 16(1): $12-25$

13. Foa EB, Hembree E, Rothbaum BO (2007) Prolonged Exposure Therapy for PTSD: Emotional Processing of Traumatic Experiences. Oxford University Press, New York.

14. Smucker MR (2005) Imagery rescripting and reprocessing therapy (IRRT): A treatment manual for adult survivors of childhood sexual abuse experiencing PTSD.International Trauma Institute, Milwaukee, United States.

15. Lee DA, Scragg P, Turner S (2001) The role of shame and guilt in traumatic events A clinical model of shame-based and guilt-based PTSD. British Journal of Medical Psychology 74(4): 451-466.

16. Wampold BE, Budge SL (2012) The 2011 Leona Tyler Award Address 
The relationship - and its relationship to the common and specific factors of psychotherapy.The Counseling Psychologist 40: 601-603.

17. Llewelyn S, Hardy G (2001) Process research in understanding and applyingpsychological therapies. British Journal of Clinical Psychology 40(1): $1-21$.

18. Timulak L (2010) Significant events in psychotherapy: An update of research findings. Psychology and Psychotherapy: Theory Research and Practice 83(4): 421-447.

19. Elliott R (1985) Helpful and nonhelpful events in brief counseling interviews: An empiricaltaxonomy. Journal of Counseling Psychology 32(3): 307-322.

20. Kazdin AE (2009) Understanding how and why psychotherapy leads to change. Psychotherapy Research 19(4-5): 418-428.
21. Carey TA, Kelly RE, Mansell W, Tai SJ (2012) What's therapeutic about the therapeutic relationship A hypothesis for practice informed by Perceptual Control Theory.The Cognitive Behaviour Therapist 5(2-3): 47-59.

22.Wells A (2009) Metacognitive therapy for anxiety and depression. GuilfordPress. New York.

23. Carey TA (2011) Exposure and reorganization: The what and how of effective psychotherapy. Clinical Psychology Review, 31(2): 236-248.

24. Webb CA, DeRubeis RJ, Amsterdam JD, Shelton RC, Hollon SD, et al. (2011) Two aspects of the therapeutic alliance: Differential relations with depressive symptom change. Journal of Consulting and Clinical Psychology 79(3): 279-283. 